\title{
Morphometric Study of the Human Brainstem and Its Neurovascular Relations
}

\author{
Bianca Aurora SZABO ${ }^{1,2^{*}}$, Raluca PASCALAU ${ }^{3^{*}}$, Vlad Adrian PADUREAN ${ }^{3^{*}}$ \\ ${ }^{1}$ Iuliu Hatieganu University of Medicine and Pharmacy, Department of Anatomy and Embryology, Cluj-Napoca, Romania \\ ${ }^{2}$ Emergency County Hospital Cluj, Department of Ophthalmology, Cluj-Napoca, Romania \\ ${ }^{3}$ Iuliu Hatieganu University of Medicine and Pharmacy, Faculty of Medicine, Cluj-Napoca, Romania \\ *Drs Szabo, Pascalau and Padurean have contributed equally to this study and should be considered to be main authors.
}

\section{ABSTRACT}

AIM: To provide a concise description of the external configuration, its internal correspondence and the neurovascular relations of the brainstem with morphometric data that can be useful in surgical planning.

MATERIAL and METHODS: The study was conducted on formalin fixed brainstem specimens, which were harvested with respect to the topographic anatomy. Macroscopic measurements were performed with a Vernier caliper.

RESULTS: The anatomical structures on the ventral and dorsal aspects of the brainstem are described and illustrated schematically. Their dimensions are also graphically represented using the mean values. Serial axial sections through the brainstem demonstrate the internal correspondence of its external features. Then, the apparent origins and the proximal diameters of the cranial nerves and the arteries of the posterior circulation as well as their anatomical relations are presented.

CONCLUSION: The external morphometry of the brainstem correlated with the position of the internal structures provides landmarks with applicability in neurosurgery especially in the fields of intrinsic brainstem lesions, posterior fossa and fourth ventricle surgery.

KEYWORDS: Brainstem, Cranial nerve, Rhomboid fossa, Vertebro-basilar system

\section{INTRODUCTION}

$\mathrm{T}$ The human brainstem is a vital anatomical structure containing numerous functionally essential nuclei and fibers, displayed in an intricate manner within a very small volume (1). As a result, surgery for intrinsic brainstem lesions, such as cavernous malformations or gliomas, represents a real challenge and can be followed by a large diversity of complications $(2,3)$. The precise knowledge of the external configuration of the brainstem plays an important role in surgical planning (5), because it is well known that there is a correspondence between the internal structures and the anatomical landmarks on the surface of the brainstem (15). The morphology of the posterior features of the brainstem is also important in surgery for the fourth ventricle tumors. The fourth ventricle can be approached using the transvermian or the telovelar route (4). The telovelar route is optimal because it does not involve damage of critical structures (10). However, it requires good knowledge of the anatomy of the rhomboid fossa and cerebellar peduncles (13).

In addition to the external configuration of the brainstem, it is important to know the neurovascular relations of the brainstem. The posterior fossa has an abundance of critical and fragile structures, including the cranial nerves and the posterior circulation arteries (7). Therefore, more than one third of surgeries in this region will have complications such as new neurological morbidity, posterior fossa syndrome, cerebrospinal fluid fistula, meningitis, or infection (4). 
All this considered, the aim of this paper is to provide a clear description of the external configuration and the neurovascular relations of the brainstem, documented with morphometric data, as a support for imaging-based surgical planning for brainstem and posterior fossa surgery.

\section{MATERIAL and METHODS}

The present study was conducted on ten formalin-fixed brain specimens held for teaching purposes in the anatomy collection of the luliu Hatieganu University of Medicine and Pharmacy, Cluj-Napoca, Romania, in accordance with the provisions of the Declaration of Helsinki 1995 (as revised in Edinburgh 2000). The research protocol was approved by the Ethics Committee of the luliu Hatieganu University of Medicine and Pharmacy, Cluj-Napoca, Romania.

First, the proximal segments of the cerebral arteries were dissected under a surgical microscope and their diameters and lengths were measured using a Vernier caliper. We then identified and measured the positions of the apparent origins of the cranial nerves III-XII, except for the apparent origin of the trochlear nerve (cranial nerve IV), which was not accessible. Next, we prepared eight specimens for sectioning of the cerebral and cerebellar peduncles to gain access to the dorsal surface of the brainstem. The basilar artery was cut between the origins of the superior cerebellar artery and the posterior cerebral artery. The posterior cerebral artery was sectioned distal to the origin of the posterior communicating artery. The arachnoid mater and the veins from the interpeduncular, crural, and ambient cisterns were removed taking care not to damage the oculomotor and trochlear nerves or the pineal gland. Next, the mesencephalon was horizontally sectioned with a brain sectioning knife.

To prepare the cerebellar peduncles, the cerebellar arteries were sectioned approximately two to three centimeters distal to their origins, and the arachnoid mater, along with the small vessels from the cerebellomesencephalic, cerebellopontine, and cerebellomedullary fissures, was removed while preserving the cranial nerves V-VIII. Next, a surgical scalpel was used for an oblique cut starting in the depth of the cerebellomesencephalic fissure, continuing laterally and inferiorly through the superior and the upper half of the middle cerebellar peduncles. The inferior cerebellar peduncle and the lower half of the middle cerebellar peduncle were sectioned by cutting laterally and superiorly from the cerebellomedullary fissure until the two oblique cuts joined each other.

Now that the brainstem was completely separated from the other parts of the cerebrum, the position of the apparent origin of the cranial nerve IV could be measured.

Morphometry of the external configuration of the brainstem was performed using a Vernier caliper. To access the rhomboid fossa, the inferior medullary velum was removed and the superior medullary velum was transformed into a flap by cutting the medial margin of the superior cerebellar peduncle and superiorly reflecting it. In the last stage of the process, 4-mm thick axial sections were prepared from the specimens using the brain sectioning knife.
Digital photographs were taken using a Nikon COOLPIX L120 camera and artwork was created using Corel PHOTO-PAINT 9 software.

\section{- RESULTS}

The external configuration of the human brainstem can be compared with the surface of a cylinder. To facilitate the description, we will divide the surface into an anterolateral or ventral surface and a dorsal surface. The most important structures on these faces and the mean values of their dimensions will be presented followed by axial sections illustrating their internal correspondence. In addition, the mean diameters of the cranial nerves and the posterior circulation arteries, as well as their position related to landmarks on the surface of the brainstem, will be described.

\section{Ventral Surface}

The ventral surface of the brainstem longitudinally extends 52 millimeters $(\mathrm{mm})$ from the pyramidal decussation to the horizontal line that passes through the most inferior points of the mammillary bodies (Figures 1, 2A-J). It is divided into three segments by the pontomesencephalic and pontomedullary sulci: the mesencephalon or midbrain, the pons, and the bulbus or medulla oblongata.

The ventral surface of the midbrain has a height of $6.62 \mathrm{~mm}$, and it is laterally formed by the $13.28 \mathrm{~mm}$ wide cerebral peduncles and the interpeduncular fossa, which has a triangular shape, on the midline. The apex of the triangle is located on the pontomesencephalic sulcus and the base of the triangle is tangent to the mammillary bodies.

The middle segment is the ventral face of the pons extending $26.1 \mathrm{~mm}$. The pontine sulcus, with a width of $3.37 \mathrm{~mm}$, is located on the midline. The $13.48 \mathrm{~mm}$ wide pontine pyramids, which continue further laterally with the middle cerebellar peduncles, are found lateral to the pontine sulcus.

The medulla oblongata has a length of $19.45 \mathrm{~mm}$ and is divided into two symmetrical halves by the median sulcus, which continues into the spinal cord inferior to the pyramidal decussation. The superior two thirds of the bulbus differ from the inferior one third due to the presence of the $11.54 \mathrm{~mm}$ long and $4.9 \mathrm{~mm}$ wide olives. The most superior point of the olives is located $1.94 \mathrm{~mm}$ inferior to the pontomedullary sulcus. Their margins form the preolivary and retroolivary sulci, which unite on the inferior third of the medulla to form the anterior collateral sulcus. The $5.14 \mathrm{~mm}$ wide surfaces delineated by the median and preolivary sulci are known as the bulbar pyramids.

\section{Dorsal Surface}

The dorsal surface of the brainstem has an intraventricular portion, the rhomboid fossa, which is the floor of the fourth ventricle, and an extraventricular portion represented by the tectal plate and the cerebellar peduncles (Figures 3, 4A-H).

The rhomboid fossa has the shape of a diamond and is delineated by the internal margins of the superior and inferior cerebellar peduncles. The lateral angles of the rhombus are closed by the middle cerebellar peduncles, the inferior angle 
is the obex, and the superior corner is marked by the frenulum of the superior medullary velum of the fourth ventricle. Its longitudinal diagonal is $32.3 \mathrm{~mm}$ long and corresponds to the median fissure. The transversal diagonal is $15.05 \mathrm{~mm}$ and is occupied by the acoustic stria, which divides the rhomboid fossa in two triangles. The most important structures visible in the upper triangle, within the pons, are the facial colliculi paramedially located at the base of the triangle. They are $4.25 \mathrm{~mm}$ wide and continue superiorly with the $3.4 \mathrm{~mm}$ wide median eminences delineated by sulcus limitans. In the lower or bulbar triangle, there are three areas known as wings: the internal white wing spreads $2.81 \mathrm{~mm}$ on each side of the median fissure; lateral to the internal white wing is the gray wing with a width of $3.33 \mathrm{~mm}$, and most laterally is the external white wing measuring $4.63 \mathrm{~mm}$.

Apart from the rhomboid fossa, the dorsal surface of the brainstem is represented by the dorsal midbrain and the cerebellar peduncles. The quadrigeminal colliculi or the tectal plate is located on the dorsal surface of the midbrain. The

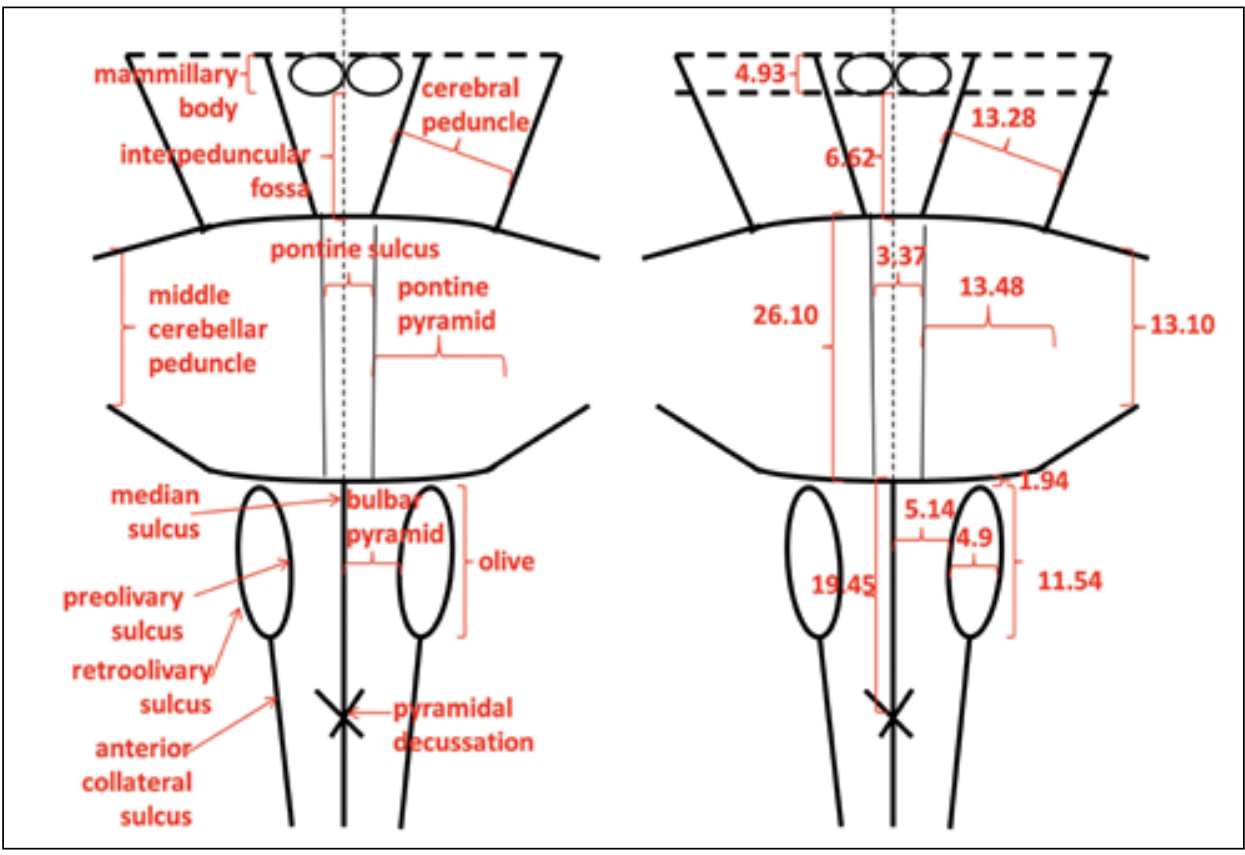

Figure 1: Schematic drawing and morphometry of the ventral surface of the brainstem.

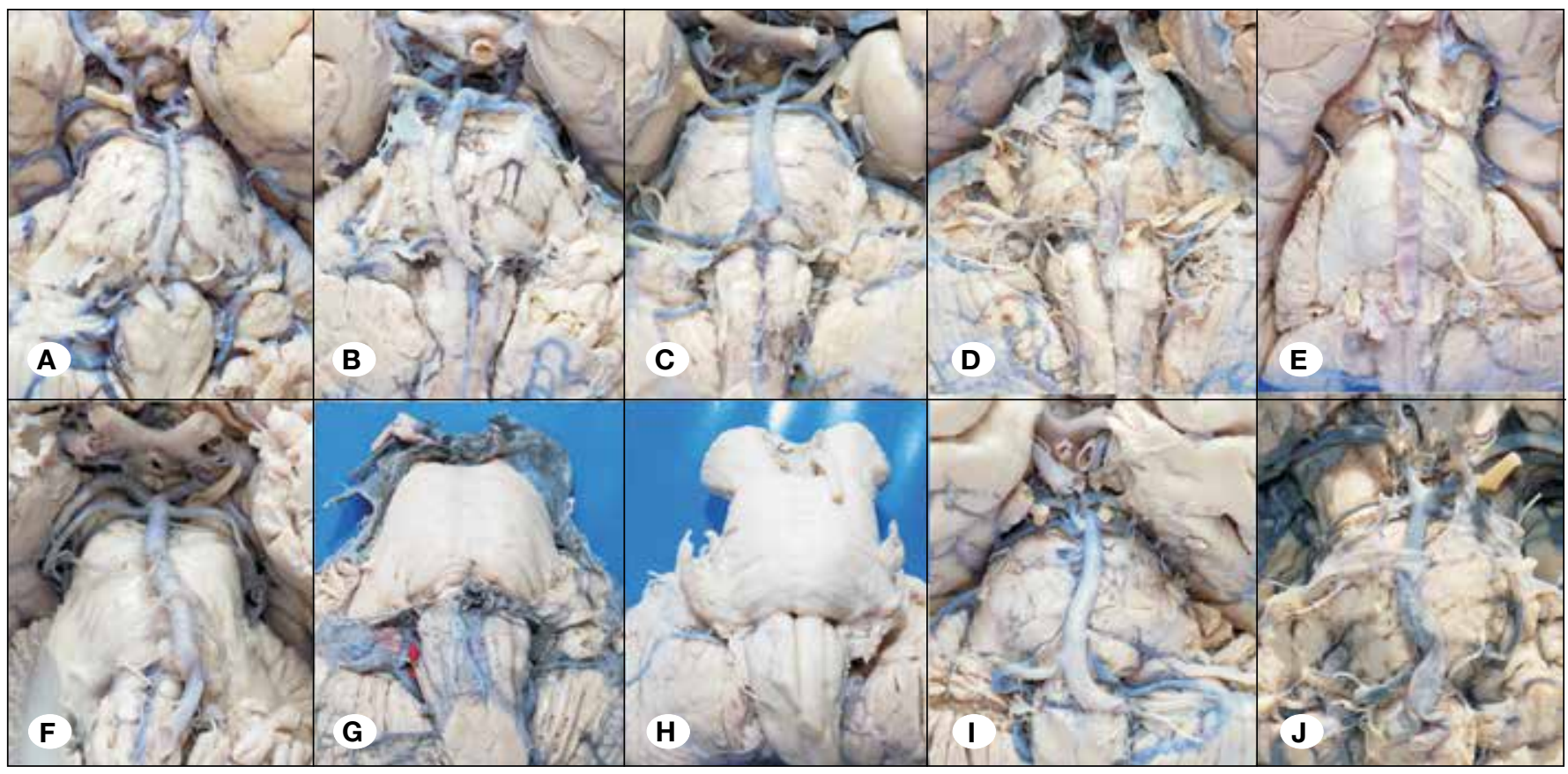

Figure 2: The ventral surface of the brainstem. 


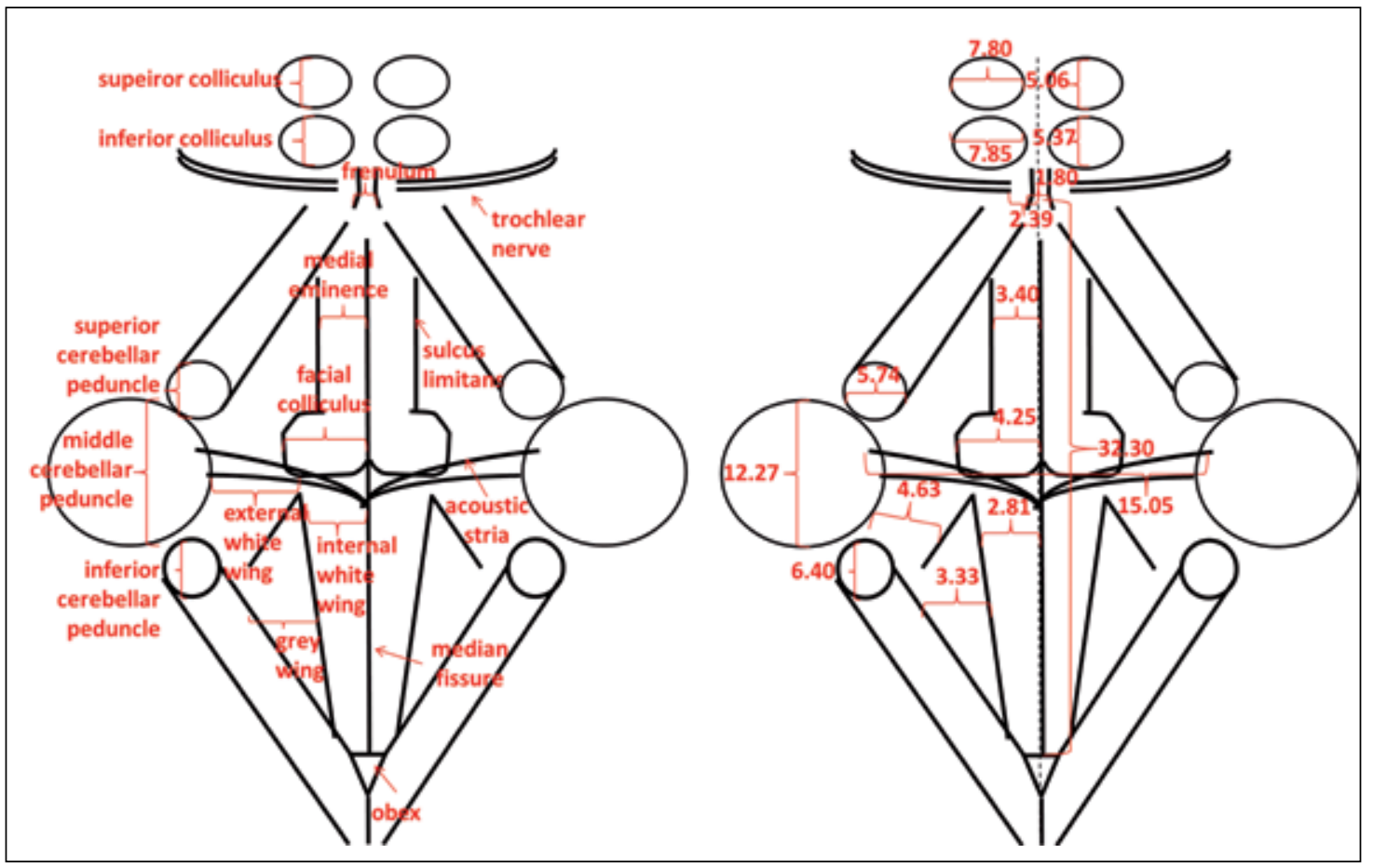

Figure 3: Schematic drawing and morphometry of the dorsal surface of the brainstem.

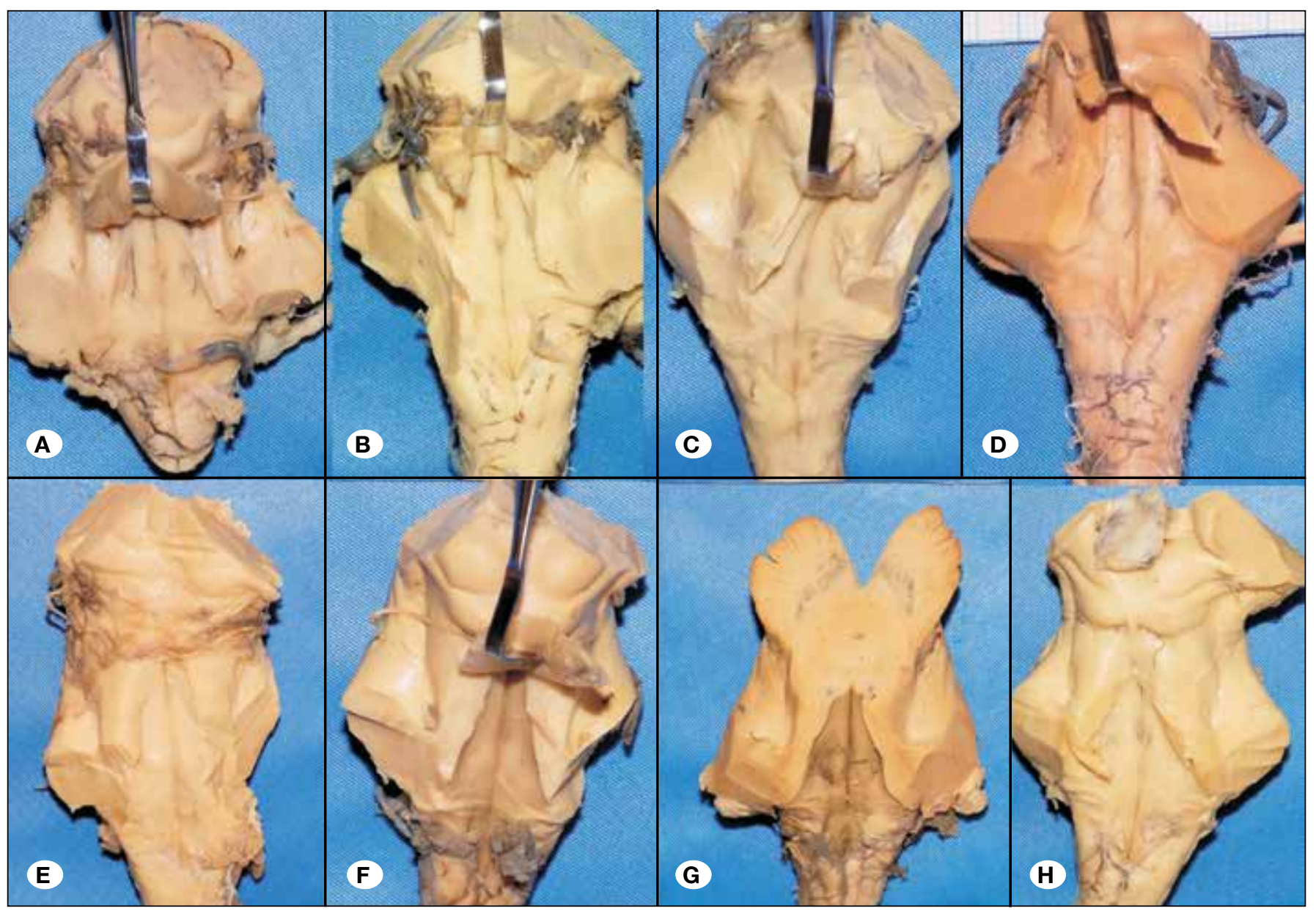

Figure 4: The dorsal surface of the brainstem. 
superior colliculi have a craniocaudal diameter of $5.06 \mathrm{~mm}$ and a transversal diameter of $7.8 \mathrm{~mm}$, and the inferior colliculi have diameters of $5.37 \mathrm{~mm}$ and $7.85 \mathrm{~mm}$, respectively. Inferior to the inferior colliculi, on the midline, there is a $1.8 \mathrm{~mm}$ wide band named frenulum of the superior medullary vellum. The superior, middle, and inferior cerebellar peduncles are located lateral to the rhomboid fossa and have maximal diameters of $5.74 \mathrm{~mm}, 12.27 \mathrm{~mm}$, and $6.4 \mathrm{~mm}$, respectively.

\section{Internal Correspondence of the External Structures}

The correspondence between the external landmarks and the inner structures of the brainstem can be observed on the axial sections (Figure 5). From the structures described on the ventral aspect, the most relevant ones are the cerebral peduncles, the pontine pyramids, and the bulbar pyramids, which are the projections of the corticonuclear and corticospinal tracts. On the dorsal aspect of the brainstem, the superior and inferior colliculi are important nuclei involved in the visual and auditory pathways, and they correspond to the deeply situated nuclei of cranial nerves III and IV, respectively. In the pontine triangle, the facial colliculus is the projection of the motor nucleus of the abducens nerve and the intraneuraxial fibers of the facial nerve looping around, and the median eminence corresponds to the medial longitudinal fasciculus. The medullary triangle consists of three areas that run from lateral to medial: the external white wing, which is the protrusion of the medial vestibular nucleus, the gray wing or the vagal trigone corresponding to the vagal nucleus, and the internal white wing or the hypoglossal trigone, which is the projection of the hypoglossal nucleus.

\section{Apparent Origin of the Cranial Nerves}

By apparent origin, we understand the place where the cranial nerves III to XII leave or enter the brainstem. Cranial nerves I and II have no relation to the brainstem and will not be described in this paper. Except for the trochlear nerve, the cranial nerves have their apparent origin on the ventral surface of the brainstem in a craniocaudal order according to their number (Figure 5).

Cranial nerve III (oculomotor nerve) has an origin in the interpeduncular fossa $1.97 \mathrm{~mm}$ lateral to the midline and $1.95 \mathrm{~mm}$ above the pontomesencephalic sulcus. Its diameter is $2.59 \mathrm{~mm}$. The origin of the cranial nerve IV, the trochlear nerve, is on the posterior surface of the mesencephalon (Figure 3) $2.39 \mathrm{~mm}$ lateral to the midline. It is the thinnest cranial nerve with a diameter of only $0.86 \mathrm{~mm}$ as opposed to the trigeminal nerve (cranial nerve $\mathrm{V}$ ), which has a diameter of $4.56 \mathrm{~mm}$ at its apparent origin on the ventral surface of the pons $15.12 \mathrm{~mm}$ lateral to the midline, $9.97 \mathrm{~mm}$ inferior to the pontomesencephalic sulcus, and $10.85 \mathrm{~mm}$ superior to the pontomedullary sulcus. The abducens nerve (cranial nerve $\mathrm{VI}$ ) has an origin in the pontomedullary sulcus $3.24 \mathrm{~mm}$ lateral to the midline above the bulbar pyramid, and measures

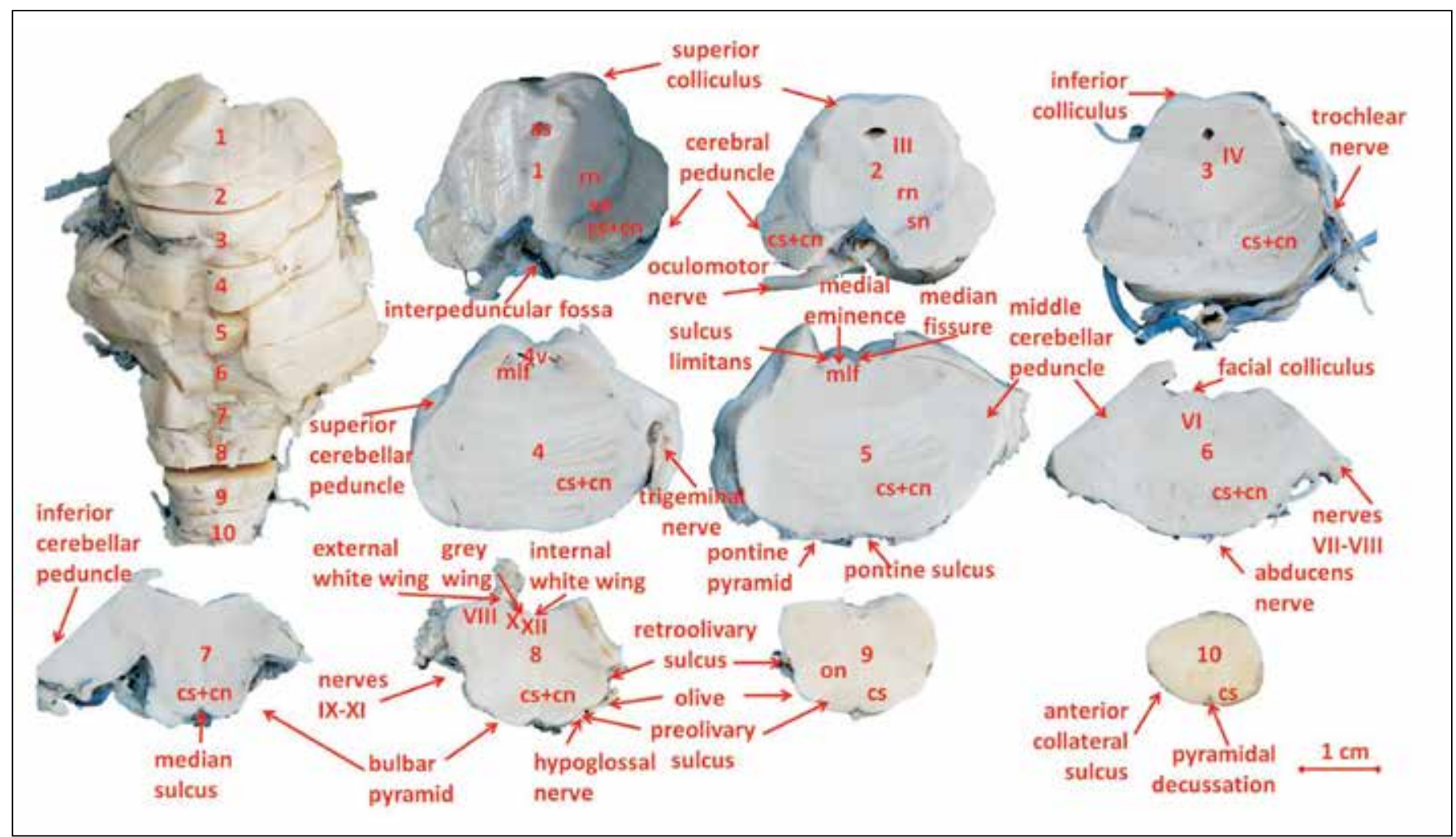

Figure 5: Axial sections through the brainstem. cs + cn = corticospinal and corticonuclear tracts; III = oculomotor nucleus; IV = trochlear nucleus; $\mathbf{r n}=$ red nucleus; $\mathbf{s n}=$ substantia nigra; $\mathbf{a s}=$ aqua ductus Sylvius; $\mathbf{4} \mathbf{v}=$ fourth ventricle; $\mathbf{V I}=$ abducens nucleus; $\mathbf{m l f}=$ medial longitudinal fasciculus; VII = medial vestibular nucleus; $\mathbf{X}=$ vagal nucleus; $\mathbf{X I}=$ hypoglossal nucleus; on = olivary nuclei. 
$1.91 \mathrm{~mm}$. Cranial nerve VII (facial nerve) also originates in the pontomedullary sulcus above the olives, $11.48 \mathrm{~mm}$ from the midline. It has a diameter of $2.48 \mathrm{~mm}$ and forms a complex with the intermediary (VII*) and vestibulocochlear (VIII) nerves, which originate lateral to cranial nerve VII, at $15.17 \mathrm{~mm}$. The rootlets of these last two nerves are grouped and measure $3.13 \mathrm{~mm}$. The apparent origins of cranial nerves IX to XI, glossopharyngeal, vagus, and accessory, respectively, are located in the retroolivary sulcus. The most superior rootlet of cranial nerve IX is $3.8 \mathrm{~mm}$ inferior to the pontomedullary sulcus, and the distance from it to the most inferior rootlet of cranial nerve $\mathrm{XI}$ is of $8.74 \mathrm{~mm}$. Similarly, the hypoglossal nerve or cranial nerve XII has an apparent origin as multiple rootlets in the preolivary sulcus. The most superior is situated $4.67 \mathrm{~mm}$ below the pontomedullary sulcus and the distance between the upper and the lower rootlets is $9.81 \mathrm{~mm}$ (Figure 6).

\section{Arteries}

The arteries of the posterior circulation, namely the vertebral artery, the basilar artery, the posterior cerebral artery, and the three pairs of cerebellar arteries are associated with the brainstem (Figure 7). They are located on the ventral surface of the brainstem.

Only the most distal segment of the vertebral artery is associated with the brainstem and is located anterior to the bulbar pyramids and medial to the rootlets of the hypoglossal nerve. Before joining to form the basilar artery at the level of the pontomedullary sulcus, the two distal vertebral arteries each have diameters of $3.66 \mathrm{~mm}$. From its origin to its end at the level of the interpeduncular fossa, the basilar artery measures $26.48 \mathrm{~mm}$. It occupies the pontine sulcus and has a caliber of $4.29 \mathrm{~mm}$. The oculomotor and abducens nerves pass just lateral to it. The posterior cerebral artery emerges from the basilar artery in the interpeduncular fossa and has a diameter of $2.59 \mathrm{~mm}$. It encircles the cerebral peduncle crossing the superior face of the oculomotor nerve.

The superior cerebellar artery originates from the basilar artery $1.82 \mathrm{~mm}$ inferior to the posterior cerebral artery, at the level of the pontomesencephalic sulcus, and passes underneath cranial nerve III and above cranial nerves IV and V to the superior surface of the cerebellum. With a diameter of $1.82 \mathrm{~mm}$, it is also associated with the superior and middle cerebellar nuclei. The anteroinferior cerebellar artery emerges from the basilar artery $16.02 \mathrm{~mm}$ inferior to the superior cerebellar artery on the ventral surface of the pons. It has a diameter of $1.36 \mathrm{~mm}$ and crosses the pontine pyramid superior to the abducens nerve, passes between cerebral nerves VII and VIII, and continues its trajectory on the inferior margin of the middle cerebellar peduncle. The posteroinferior cerebellar artery originates from the vertebral artery just inferior to the pontomedullary sulcus, and $10.8 \mathrm{~mm}$ inferior to the anteroinferior cerebellar artery. Its proximal diameter measures $1.95 \mathrm{~mm}$, and it has a tortuous course to the inferior surface of the cerebellum.

\section{DISCUSSION}

Tumors and vascular malformations located in the brainstem were considered unresectable for a long time due to the high risk of complications (5). In the past few decades, the advances in neuroimaging and neurophysiological monitoring corroborated with anatomical knowledge and the experience of neurosurgeons have led to the refining of the surgical approaches to lesions located in or around the brainstem $(1,3,15)$.

In this paper, the location of some anatomical structures on the surface of the brainstem is described according to landmarks that are easy to identify intraoperatively or on imaging studies.

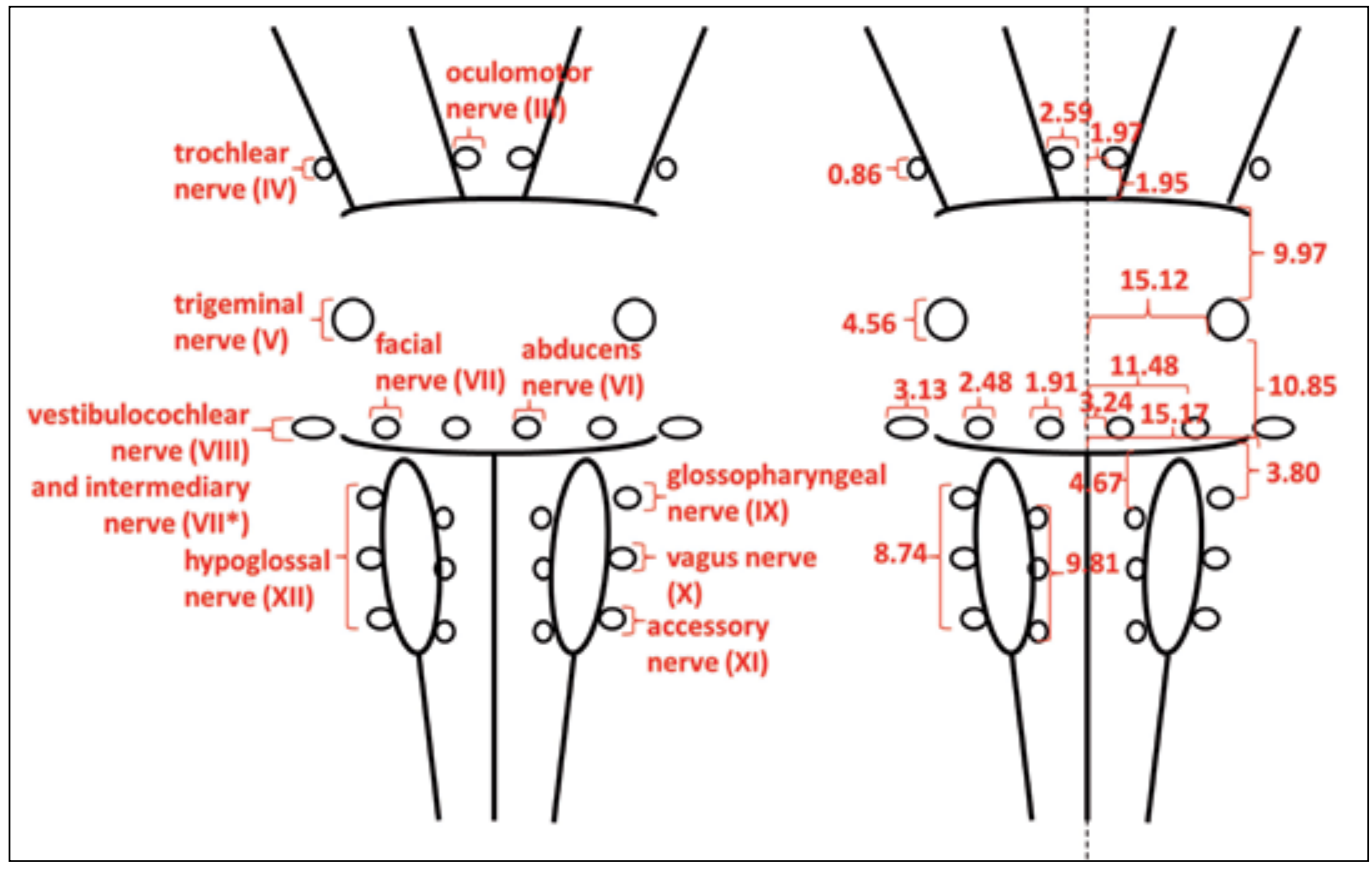

Figure 6: Schematic drawing and morphometry of the apparent origin of the cranial nerves. 


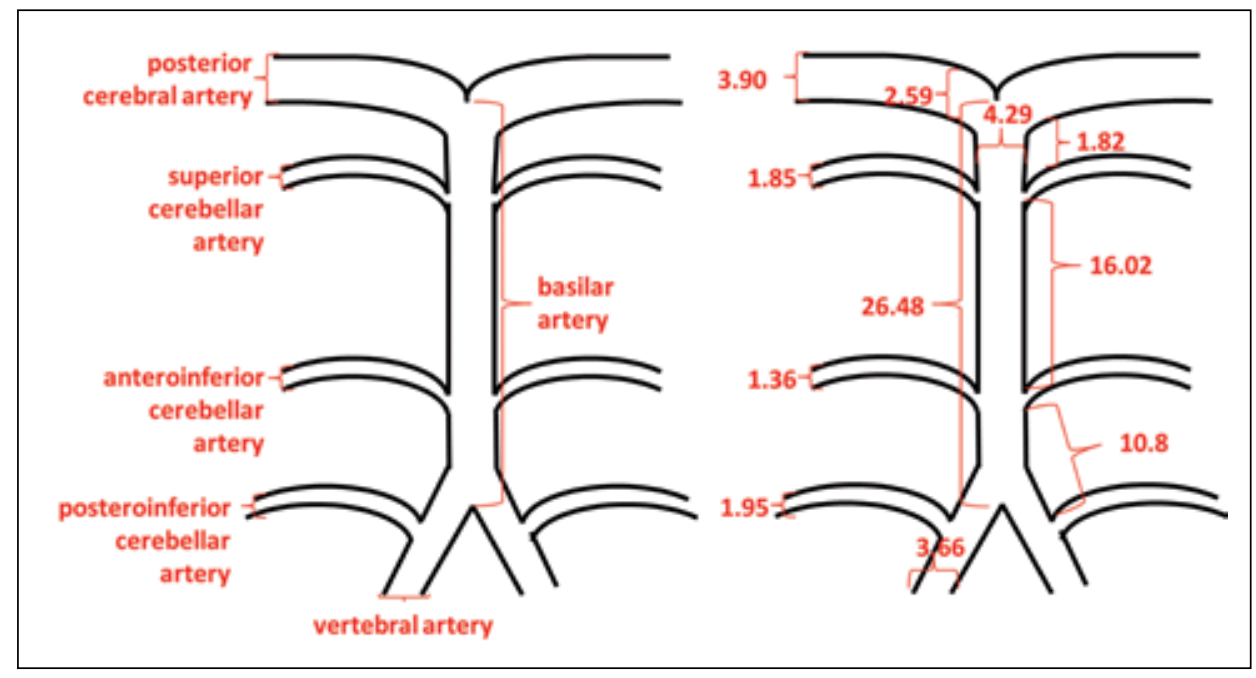

Figure 7: Schematic drawing and morphometry of the posterior circulation arteries.

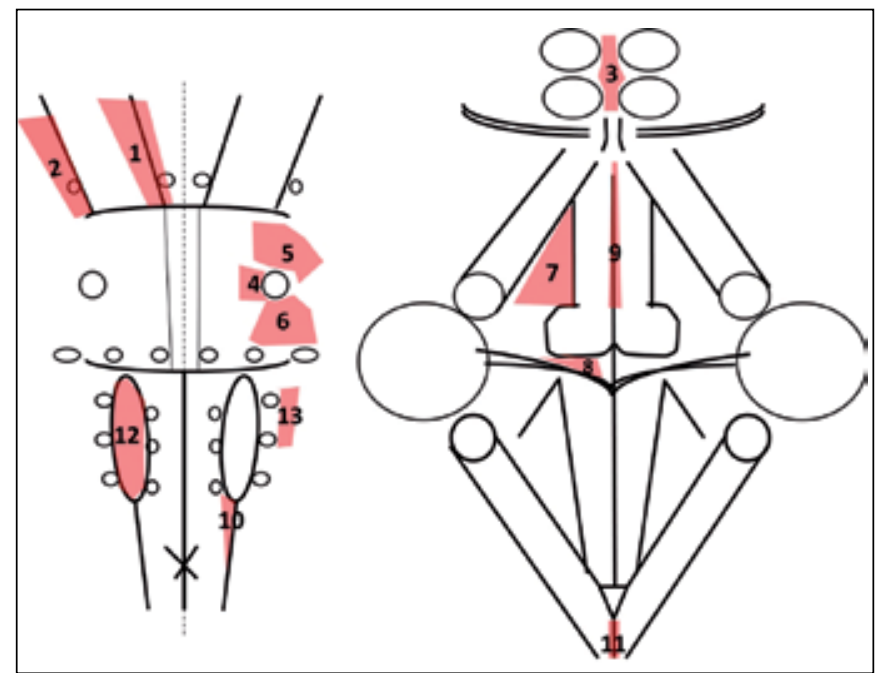

Figure 8: Safe entry zones according to Cavalcanti et al. (1). 1) anterior mesencephalic; 2) lateral mesencephalic; 3) intercollicular; 4) peritrigeminal; 5) supratrigeminal; 6) lateral pontine; 7) supracollicular; 8) infracollicular; 9) median sulcus of the fourth ventricle; 10) anterolateral sulcus of medulla; 11) posterior median sulcus of medulla; 12) olivary zone; 13) lateral medullary zone.

On the ventral surface, the pyramids correspond to the descending pathways $(11,15)$, and must not be damaged during surgery. The elements of the rhomboid fossa are of particular importance because they must be considered during not only intrinsic brainstem lesion surgery (1) but also fourth ventricle surgery (10). Based on the correspondence between the external configuration of the brainstem and the internal location of the critical structures, at least thirteen safe entry zones on the surface of the brainstem (1), which are defined as small areas where the brainstem can be incised to approach intrinsic brainstem lesions without injuring critical structures and without encountering perforating arteries (15), have been described. However, large lesions can distort the normal anatomy, and the anatomical landmarks on the surface will no longer lead to the safe entry zones (3). In this context, knowing the normal distances between the apparent origins of the cranial nerves and the midline, as they are reported in this study, will help assess the distortions in preoperative imaging studies. According to Cavalcanti et al. (1), the location of the safe entry zones in relation to the external anatomy of the brainstem presented in our study is illustrated in Figure 8.

The neurovascular associations of the brainstem are essential in posterior fossa surgery (12). In this type of surgery, cranial nerves are especially at risk (17), due to direct manipulation or ischemia as a consequence of injuring the regional arteries that supply these nerves (6). In addition, neurovascular conflicts can occur due to contacts between the arteries of the posterior circulation and the cranial nerves (16). In these cases, the associations between the arteries and the cranial nerves that are presented in this paper are essential for the diagnosis of neurovascular conflicts and the treatment, which consists of microvascular decompression (14). For example, the association between the trigeminal nerve and the superior cerebellar or the anteroinferior cerebellar artery can be a cause of trigeminal neuralgia $(8,14)$. The anteroinferior cerebellar artery is also associated with the facial nerve and a neurovascular conflict between them can be documented in cases of hemifacial spasm (9).

\section{CONCLUSION}

The present study was based on the morphometric assessment of the external anatomy of the brainstem and its neurovascular relations, namely cranial nerves III-XII and the arteries of the posterior circulation. It was conducted on formalin-fixed brainstem specimens, which were isolated with respect to the anatomy by sectioning the cerebral and cerebellar peduncles. The results have applicability in neurosurgery especially in the fields of intrinsic brainstem lesions, posterior fossa, and fourth ventricle surgery. 


\section{ACKNOWLEDGEMENT}

This research was carried out with funding from "Iuliu Hatieganu" University of Medicine and Pharmacy, Dept. of Anatomy and Embryology, Cluj-Napoca, Romania, internal grant no. 4995/17/08.03.2016.

\section{REFERENCES}

1. Cavalcanti DD, Preul MC, Kalani YS, Spetzler RF: Microsurgical anatomy of safe entry zones to the brainstem. J Neurosurg 124: 1359-1376, 2016

2. Cavalheiro S, Yagmurlu K, Silva da Costa MD, Mendonça Nicácio J, Pereira Rodrigues T, Chaddad-Neto F, Rhoton AL: Surgical approaches for brainstem tumors in pediatric patients. Childs Nerv Syst 31:1815-1840, 2015

3. Deletis V, Fernández-Conejero I: Intraoperative monitoring and mapping of the functional integrity of the brainstem. Clin Neurol 12: 262-273, 2016

4. Ezer H, Banerjee AD, Bollam P, Guthikonda B, Nanda A: The superior transvelar approach to the fourth ventricle and brainstem. J Neurol Surg B 73:175-182, 2012

5. Fard SA, Adeeb N, Rezaei M, Kateb B, Mortazavi MM: Resection of deep brain stem lesions: Evolution of modern surgical techniques. JNBS 3:29-31, 2016

6. Hendrix P, Griessenauer CJ, Foreman P, Loukas M, Fisher WS, Rizk E, Shoja MM, Tubbs RS: Arterial supply of the lower cranial nerves: A comprehensive review. Clin Anat 27:108117,2014

7. Isolan GR, Pereira AH, Aguiar PH, Antunes ÁC, Mousquer JP, Pierobon MR: Microsurgical anatomy of the infratentorial arteries: Stereoscopic printing study. J Vasc Bras 11:114-122, 2012
8. Krzyżewski RM, Stachura MK, Stachura AM, Rybus J, Tomaszewski KA, Klimek-Piotrowska W, Brzegowy P, Urbanik A, Walocha JA: Variations and morphometric analysis of the proximal segment of the superior cerebellar artery, Neurol Neurochir Pol 48:229-235, 2014

9. Lee MS, Kim MS, Hong IS, Whank K, Han YP: Clinical usefulness of magnetic resonance cisternography in patients having hemifacial spasm. Yonsei Med J 42: 390-394, 2001

10. Liu R, Kasper EM: Bilateral telovelar approach: A safe route revisited for resections of various large fourth ventricle tumors. Surg Neurol Int 5:16, 2014

11. Middlebrooks EH, Benett JA, Bidari S, Crow A: Visualization of the pyramidal decussation utilizing diffusion tensor imaging: A feasibility study utilizing generalized q-sampling imaging. Research 1:711, 2014

12. Ono M, Rhoton AL Jr, Barry M: Microsurgical anatomy of the region of the tentorial incisura. J Neurosurg 60:365-399, 1984

13. Perrini P, Tiezzi G, Castagna M, Vannozzi R: Three-dimensional microsurgical anatomy of cerebellar peduncles. Neurosurg Rev 36: 215-224, 2013

14. Ramesh VG, Premkumar G: An anatomical study of the neurovascular relationships at the trigeminal root entry zone. J Clin Neurosci 16: 934-936, 2009

15. Recalde RJ, Figueiredo EG, de Oliveira E: Microsurgical anatomy of the safe entry zones on the anterolateral brainstem related to surgical approaches to cavernous malformations. Neurosurg 62:9-17, 2008

16. Saeki N, Rhoton A: Microsurgical anatomy of the upper basilar artery and the posterior circle of Willis. J Neurosurg 46:563578, 1977

17. Ziyal IM, Bilginer B, Ozcan OE, Basar R, Sekhar LN, Ozgen $\mathrm{T}$ : Course and relationship of cranial nerves from end organs through foraminas to root entry zones. How far can they be mobilized: An anatomical study. Neuroanatomy 3: 46-50, 2004 